\title{
Credibility-limited Functions for Belief Bases
}

\author{
EDUARDO FERMÉ, Departamento de Matemática, Universidade de \\ Madeira,Universitario de Penteada, 9000-390 Funchal, Madeira, Portugal. \\ E-mail:ferme@math.uma.pt
}

JUAN MIKALEF and JORGE TABOADA, Computer Science Department, University of Buenos Aires, Argentina.

E-mail:\{juan.mikalef,jorge.taboada\}@oracle.com

\begin{abstract}
In this paper we adapt the Fermé and Hansson model of Shielded Contraction as well as Hansson et al. Credibilitylimited Revision for belief bases, to join two of the many variations of the AGM model, i.e. those in which knowledge is represented through belief bases instead of logic theories, and those in which the object of the epistemic change does not have priority over the existing information as is the case in the AGM model.

Keywords: Logic of theory change, nonprioritzed belief functions, belief bases.
\end{abstract}

\section{Introduction}

In the logic of theory change, the AGM model has acquired the status of a standard model [1]. However, the AGM model seems to be improper in many contexts. This inspired many researchers to propose extensions and generalizations to AGM. Among these extensions, we can mention the following. 1. Nonprioritized belief revision: the AGM model always accepts the new information. This feature appears, in general, to be unrealistic, since rational agents, when confronted with information that contradicts previous beliefs, often reject it altogether or accept only parts of it. The nonprioritized models allow new functions, where the new information has no special priority (see [8] for a survey of different proposals). In the case of contraction, the input sentence is always retracted (except in the limiting case of tautologies), independently of the original set of beliefs. 2. Extensions in the representation of the belief states and of the inputs: belief sets (sets of sentences closed under logical consequence) cannot distinguish between fundamental and derived beliefs. Belief bases, sets of sentences not necessarily closed under logical consequence, have been shown to possess more expressive power than belief sets [5-7, 12-14].

The objective of this paper is to present a model that combines both kinds of extensions. This model consists of the extension for belief bases of Shielded Contraction [4], in which the contraction operations are filtered by a group of sentences capable of being retracted, and Credibility-limited Revision [10] in which the new information in the revision is only added if certain acceptability conditions are fulfilled. We will enunciate the axiomatic for these models, a constructive model for each one of these operations, establishing the corresponding representation theorem. 


\section{Background}

\subsection{Formal preliminaries}

We will assume a language $\mathcal{L}$ that is closed under truth-functional operations and a consequence operator $C n$ for $\mathcal{L}$. $C n$ satisfies the standard Tarskian properties, namely inclusion ( $A \subseteq C n(A)$ ), monotony (if $A \subseteq B$, then $C n(A) \subseteq C n(B)$ ), and iteration $(C n(A)=C n(C n(A)))$. It is supraclassical and compact, and satisfies deduction (if $\beta \in C n(A \cup\{\alpha\})$, then $(\alpha \rightarrow \beta) \in C n(A))$. $A \vdash \alpha$ will be used as an alternative notation for $\alpha \in C n(A)$ and $C n(\alpha)$ for $C n(\{\alpha\})$. Upper-case letters denote subsets of $\mathcal{L}$. $\top$ is an arbitrary tautology and $\perp$ an arbitrary contradiction. A set of sentences that is closed under logical consequence is called a belief set, otherwise it is a belief base.

\subsection{Belief bases functions}

The use of logically closed sets to represent beliefs has received much criticism. Among them we can mention: 1. Belief sets make no distinction between basic beliefs and those which were inferred from them. 2. They are computability intractable. 3. Belief bases can distinguish between different inconsistent belief states.

Several authors propose the use of belief bases for logic of theory change [5-7, 12-14]. We will use the belief base theory proposed by Hansson [6].

Base expansion is simply a set union, i.e. $A+\alpha=A \cup\{\alpha\}$.

We can construct a base contraction function using the remainder sets, i.e. maximal subsets of $A$ that fail to imply $\alpha$ :

DEFINITION 2.1

[2] Let $A$ be a belief base and $\alpha$ a sentence. The set $A \perp \alpha$ (A remainder $\alpha)$ is the set of sets such that $B \in A \perp \alpha$ if and only if:

- $B \subseteq A$.

- $B \forall \alpha$.

- There is no set $B^{\prime}$ such that $B \subset B^{\prime} \subseteq A$ and $B^{\prime} \forall \alpha$.

DEFINITION 2.2

$[1,6]$ The partial meet base contraction operator on $A$ based on a selection function $\gamma$ is the operator $\sim_{\gamma}$ such that for all sentences $\alpha$ :

$$
A \sim_{\gamma} \alpha=\cap \gamma(A \perp \alpha) .
$$

Hansson characterized partial meet base contraction in terms of postulates.

THEOREM 2.3

[6] Let $A$ be a belief base. An operator $\div$ on $A$ is a partial meet contraction function for $A$ if and only if $\div$ satisfies

Success $\quad$ If $\nvdash \alpha$, then $A \div \alpha \nvdash \alpha$.

Inclusion $A \div \alpha \subseteq A$.

Relevance If $\beta \in A$ and $\beta \notin A \div \alpha$ then there is some set $A^{\prime}$ such that $A \div \alpha \subseteq A^{\prime} \subseteq A$ and $\alpha \notin C n\left(A^{\prime}\right)$ but $\alpha \in C n\left(A^{\prime} \cup\{\beta\}\right)$. 
Uniformity If it holds for all subsets $A^{\prime}$ of $A$ that $\alpha \in C n\left(A^{\prime}\right)$ if and only if $\beta \in C n\left(A^{\prime}\right)$, then $A \div \alpha=A \div \beta$.

Partial meet base contraction also satisfies other interesting properties as we will see in the following observation:

OBSERVATION 2.4

[8] Let $A$ be a belief base and $\div$ an operator on $A$. Then:

1. If $\div$ satisfies inclusion and relevance, then it satisfies vacuity (If $A \not \forall \alpha$, then $A \div \alpha=A$ ).

2. If $\div$ satisfies uniformity, then it satisfies extensionality (If $\vdash \alpha \leftrightarrow \beta$, then $A \div \alpha=$ $A \div \beta$ )

3. If $\div$ satisfies inclusion and relevance, then it satisfies failure (If $\vdash \alpha$, then $A \div \alpha=A$ ).

In belief bases, as in belief sets, revising by $\alpha$ can be reduced to contraction by $\neg \alpha$ and then expanded by $\alpha$ (Levi identity).

\section{DEFINITION 2.5}

$[1,6]$ Let $A$ be a belief base. The partial meet base revision operator on $A$ based on a selection function $\gamma$ is the operator $* \gamma$ such that for all sentences $\alpha$ :

$$
A * \gamma \alpha=(\cap \gamma(A \perp \alpha)) \cup\{\alpha\} .
$$

The axiomatic characterization of partial meet base revision is:

THEOREM 2.6

[6] Let $A$ be a belief set. An operator $*$ on $A$ is a partial meet revision function for $A$ if and only if $*$ satisfies:

Success $\quad \alpha \in A * \alpha$.

Consistency $A * \alpha$ is consistent if $\alpha$ is consistent.

Inclusion $A * \alpha \subseteq A \cup\{\alpha\}$.

Relevance If $\beta \in A$ and $\beta \notin A * \alpha$, then there is some $A^{\prime}$ such that $A * \alpha \subseteq A^{\prime} \subseteq$ $A \cup\{\alpha\}, A^{\prime} \forall \perp$ but $A^{\prime} \cup\{\beta\} \vdash \perp$.

Uniformity If for all subsets $A^{\prime} \subseteq A, A \cup\{\alpha\} \vdash \perp$ if and only if $A^{\prime} \cup\{\beta\} \vdash \perp$, then $A \cap(A * \alpha)=A \cap(\bar{A} * \beta)$.

\section{Shielded base contraction}

In standard accounts of belief contraction, the contraction is always successful (except for the limiting case of tautologies). However, this is not intuitive in general. A rational agent can have certain nontautological beliefs which it is not disposed to resign. In the case of human agents, it is easy to find examples. In computer systems, there are integrity constraints that cannot be eliminated in the update/correction process.

The basic idea of Shielded Contraction is to define a contraction function that consists of two different steps. The first one evaluates if the input sentence is suitable to be contracted. The second one contracts in the positive case, applying a partial meet base contraction function, otherwise no change is made.

The procedure to evaluate if a given sentence can be contracted is checking if it belongs to a set of sentences denominated a retractible set, whose properties are defined in the next subsection. 


\section{Credibility-limited Functions for Belief Bases}

\subsection{The set of retractible sentences}

The set $R$ defines which sentences can be discarded from a belief base via a contraction function. The sentences in $R$ are called 'retractible' sentences and the rest 'irretractible' sentences. The construction of the retractible set is subjective and depends on how 'important' the beliefs are for an rational agent. However, we can define some rationality criteria about this set, those that will characterize the retractible sets.

The tautologies cannot be removed from the consequences of a belief base, hence all the tautologies are 'nonretractible' sentences.

\section{Nonretractability of tautology $R \cap C n(\emptyset)=\emptyset$.}

We can strengthen this property in the following way: if a sentence $\alpha$ is irretractible, then $\alpha$ cannot be eliminated from the consequence of a belief base. Hence all the consequences of $\alpha$ cannot be retracted:

Nonretractability of propagation If $\alpha \notin R$, then $C n(\alpha) \cap R=\emptyset$.

Since a conjunctive sentence $\alpha \wedge \beta$ can be removed from a belief base only if at least one of its conjuncts $\alpha$ and $\beta$ is removed, we should expect $\alpha \wedge \beta$ not to be retractible without either $\alpha$ or $\beta$ being so.

Conjunctive completeness If $\alpha \wedge \beta \in R$, then $\alpha \in R$, or $\beta \in R$.

The concept of 'irretractibility' means that a sentence cannot be removed from a belief base $A$, independently of which contraction we perform, hence:

\section{Nonretractability of preservation $\mathcal{L} \backslash R \subseteq C n(A \sim \alpha)$}

where $\sim$ is a base contraction function. Now we can define the a base contraction function using a retractible set.

DEFINITION 3.1

Let $\div$ be a partial meet base contraction on a belief base $A$. Let $R$ be the associate retractible set. Then $\sim$ is a shielded base contraction function if and only if:

$$
A \sim \alpha=\left\{\begin{array}{cl}
A \div \alpha & \text { if } \alpha \in R \\
A & \text { otherwise }
\end{array}\right.
$$

\subsection{Postulates for shielded base contraction}

Since the success postulate is given up, we must find weaker versions of it that capture our intuitions. When a sentence is irretractible (and then, impossible to contract), the contraction must return the original set of sentences: ${ }^{1}$

\footnotetext{
${ }^{1}$ Note that in the AGM model this intuition is captured by the failure postulate, since in this model the tautologies are the only irretractible sentences.
} 
Relative success $A \sim \alpha=A$ or $\alpha \notin C n(A \sim \alpha)$.

The 'status' of irrevocability is associated to a sentence per se. Then if a sentence $\beta$ cannot be removed in a contraction by itself, then it cannot be removed at all from the belief base.

Persistence If $\beta \in C n(A \sim \beta)$, then $\beta \in C n(A \sim \alpha)$.

In contraction, no sentences are added.

Inclusion $A \sim \alpha \subseteq A$.

In Shielded Base Contraction, a sentence $\beta$ is retracted in a contraction from $A$ by $\alpha$ if this sentence contributes in some way to imply $\alpha$ from $A$. Consequently we can use the same postulate mentioned in Theorem 2.3.

Relevance If $\beta \in A$ and $\beta \notin A \sim \alpha$ then there is some set $A^{\prime}$ such that $A \sim \alpha \subseteq A^{\prime} \subseteq A$ and $\alpha \notin C n\left(A^{\prime}\right)$ but $\alpha \in C n\left(A^{\prime} \cup\{\beta\}\right)$.

The postulate of success indicates the behaviour of a contraction function when a sentence is included in the consequence of a belief base, but does not determine the behaviour when the sentence to be contracted is not a logical consequence from the belief base. Then the minimal change to contract the sentence is to do nothing.

Vacuity If $A \nvdash \alpha$, then $A \sim \alpha=A$.

A natural requirement is that equivalent sentences must return the same contraction (If $\vdash \alpha \leftrightarrow \beta$, then $A \sim \alpha=A \sim \beta$ (extensionality)). In belief bases, moreover, there are other cases in which two sentences must return the same contraction. If every subset of sentences of $A$ accepts a sentence $\alpha$ if and only if it accepts a sentence $\beta$, then both sentences are indistinguishable in the contraction operation, since the reasons to accept $\alpha$ are exactly the same as the ones to accept $\beta$.

Then it is reasonable that contracting by $\alpha$ must be equivalent to contracting by $\beta$.

Uniformity If it holds for all subsets $A^{\prime}$ of $A$ that $\alpha \in C n\left(A^{\prime}\right)$ if and only if $\beta \in C n\left(A^{\prime}\right)$, then $A \sim \alpha=A \sim \beta$.

If two sentences $\alpha$ and $\beta$ are both irretractible, then we should expect their conjunction to also be irretractible.

Conjunctive constancy If $A \sim \alpha=A \sim \beta=A$ then $A \sim(\alpha \wedge \beta)=A$.

\subsection{Representation theorem}

The following representation theorem characterizes the shielded base contraction function.

THEOREM 3.2

Let $A$ be a belief base and $\sim$ an operation on $A$. Then the following conditions are equivalent: 


\section{Credibility-limited Functions for Belief Bases}

1. $\sim$ satisfies relative success, persistence, inclusion, relevance, vacuity, uniformity and conjunctive constancy.

2. $\sim$ is the operator of shielded base contraction induced by a partial meet base contraction operator $\div$ for $A$ and a set $R \subseteq \mathcal{L}$ that satisfies nonretractability propagation, and non retractability preservation and such that:

$$
A \sim \alpha=\left\{\begin{array}{cl}
A \div \alpha & \text { if } \alpha \in R \\
A & \text { otherwise }
\end{array}\right.
$$

\section{Credibility-limited base contraction}

In nonprioritized revision function, the success postulate is replaced by weaker postulates, that do not accept the new information in certain cases.

In this section we will focus on credibility-limited revision for belief bases, that is a particular case of the model decision+revision mentioned by Hansson in [8]. Our work is an extension of Credibility-limited Revision, that was presented for belief sets in [10]. ${ }^{2}$ Conceptually, in this kind of revision there are sentences that the agent is not supposed to accept even when revising by it. In these cases, this 'incredulity' is based in certain sentences that the agent is not supposed to rectify or discard.

In particular, in the credibility-limited model, the credible sentences, i.e. the sentences that the agents are dispose to accept are represented by a set $C$. This does not mean that they are currently accepted. The concept of being 'credible' is related with the concept of retractible mentioned in Section 3 and the relation can be expressed as:

$$
\alpha \in C \text { iff } \neg \alpha \in R \text {. }
$$

\subsection{The set of credible sentences}

The credibility-limited base function therefore consists of two steps: in the first one, the agent evaluates if the epistemic input is an element of $C$. If so, the second step is to revise the belief base by the input (in our case in the AGM style), otherwise, no change is made.

The following are desirable properties for the set $C$.

A natural requirement is that the contradictions are not credible.

Element consistency If $\alpha \in C$, then $\alpha \nvdash \perp$.

If a sentence is credible, its derived sentences must be credible too.

Single sentence closure If $\alpha \in C$, then $C n(\{\alpha\}) \subseteq C$.

If two sentences are not credible, their disjunction is also not credible.

Disjunctive completeness If $\alpha \vee \beta \in C$, then either $\alpha \in C$ or $\beta \in C$.

\footnotetext{
${ }^{2}$ Makinson provided an antecedent of Credibility-limited Revision in [11].
} 
If a sentence is not credible, then there must be reasons in the belief base to do that, i.e. If $\beta \notin C$, then $A \vdash \neg \beta$, or equivalently:

Expansive credibility If $A \not \alpha$, then $\neg \alpha \in C$.

The outcome of a revision must be credible.

Revision credibility If $\alpha \in C$, then $C n(A \circledast \alpha) \subseteq C$.

If a sentence is not credible, then its negation is always believed.

Strong revision credibility If $\beta \notin C$, then $A \circledast \alpha \vdash \neg \beta$.

If the belief base is consistent, expansive credibility can be strengthened.

LEMMA 4.1

If $A \nvdash \perp$ and $C$ satisfies expansive credibility, then $C n(A) \subseteq C$.

ProOF. Let $\alpha$ such that $A \vdash \alpha_{(1)}$ and $\alpha \notin C_{\circledast}$, then by expansive credibility $A \vdash \neg \alpha_{(2)}$. Hence by (1) and (2) $A \vdash \perp$, absurd.

Using the set $C$ we can define a credibility-limited base revision function.

DEFINITION 4.2

Let $A$ be a belief base. The operation $\circledast$ on $A$ is a credibility-limited base revision on $A$ if and only if there is an partial meet base revision $*$ on $A$ and a set $C$ of sentences such that for all sentences $\alpha$ :

$$
A \circledast \alpha= \begin{cases}A * \alpha & \text { if } \alpha \in C \\ A & \text { otherwise. }\end{cases}
$$

\subsection{Postulates for credibility-limited base revision}

As credibility-limited base revision is a nonprioritized revision function, then we must give up the success postulate. Since in the unsuccessful case no change is made, we need a postulate that reflects this fact.

Relative success $\alpha \in A \circledast \alpha$ or $A \circledast \alpha=A$.

We can not expect the outcome of the operation to be consistent in all cases, because in the unsuccessful case, the belief base remains unchanged. However, we can ensure consistency in the case of consistent belief bases.

\section{Consistency preservation If $A \not \perp$, then $A \circledast \alpha \not \vdash \perp$.}

As in AGM, we conserve the expansion function as the upper bound of the credibilitylimited base revision. This bound is reached when the input sentence is consistent with the belief base. 
Inclusion $A \circledast \alpha \subseteq A \cup\{\alpha\}$.

Vacuity If $A \nvdash \neg \alpha$, then $A \circledast \alpha \subseteq A \cup\{\alpha\}$.

A second weaker version of success is used for disjunctions. The revision of a disjunction is successful if at least one of the disjuncts can be successfully revised.

Disjunctive distribution If $(\alpha \vee \beta) \in A \circledast(\alpha \vee \beta)$, then $\alpha \in A \circledast \alpha$ or $\beta \in A \circledast \beta$.

A sentence $\beta$ can be eliminated in the revision by $\alpha$, only if this sentence contributes in some way to imply $\neg \alpha$ from $A$.

Relevance If $\beta \in A$ and $\beta \notin A \circledast \alpha$, then there is some $A^{\prime}$ such that $A * \alpha \subseteq A^{\prime} \subseteq$ $A \cup\{\alpha\}, A^{\prime} \nvdash \perp$ but $A^{\prime} \cup\{\beta\} \vdash \perp$.

If a sentence is successfully revised, then its consequences will be accepted in the revised belief base. Therefore it is intuitive to require that these sentences can also be successfully revised.

Strict improvement If $\alpha \in A \circledast \alpha$ and $\vdash \alpha \rightarrow \beta$, then $\beta \in A \circledast \beta$.

We can strengthen this postulate to all the sentences accepted in the revised belief base.

Regularity If $A \circledast \alpha \vdash \beta$, then $\beta \in A \circledast \beta$.

Strong regularity If $A \circledast \alpha \nvdash \neg \beta$, then $\beta \in A \circledast \beta$.

Note that when $A$ is consistent regularity follows from strong regularity and consistency preservation. As in contraction, we strengthen the postulate of extensionality to uniformity.

Uniformity If for all subsets $A^{\prime} \subseteq A, A^{\prime} \cup\{\alpha\} \vdash \perp$ if and only if $A^{\prime} \cup\{\beta\} \vdash \perp$, then $A \cap(A \circledast \alpha)=A \cap(A \circledast \beta)$.

\subsection{Representation theorem}

THEOREM 4.3

Let $A$ be a consistent belief base and $\circledast$ an operation on $A$. Then the following conditions are equivalent:

1. $\circledast$ satisfies relative success, consistency preservation, inclusion, vacuity, disjunctive distribution, relevance, strong regularity, and uniformity.

2. $\circledast$ is the operator of credibility-limited base revision induced by a partial meet base revision operator for $A$ and a set $C$ that satisfies element consistency, single sentence closure, disjunctive completeness, expansive credibility, revision credibility and strong revision credibility and such that

$$
A \circledast \alpha= \begin{cases}A * \alpha & \text { if } \alpha \in C \\ A & \text { otherwise. }\end{cases}
$$




\section{Acknowledgements}

We specially want to thank Renata Wassermann for her indepth review of our work. We are also indebted to Sven Ove Hansson who checked the correctness of the representation theorems and to Verónica Becher for her comments and remarks. Thanks also to Andrea Scagnetti for corrections concerning presentation.

\section{References}

[1] C. Alchourrón, P. Gärdenfors, and D. Makinson. On the logic of theory change: Partial meet contraction and revision functions. Journal of Symbolic Logic, 50, 510-530, 1985.

[2] C. Alchourrón and D. Makinson. Hierarchies of regulations and their logic. In New Studies in Deontic Logic: Norms, Actions, and the Foundations of Ethics, Risto Hilpinen, ed., pp. 125-148, 1981.

[3] C. Alchourrón and D. Makinson. On the logic of theory change: Contraction functions and their associated revision functions. Theoria, 48, 14-37, 1982.

[4] E. Fermé and S. O. Hansson. Shielded contraction. In Frontiers in Belief Revision, H. Rott and M.-A. Williams, eds, pp. 85-107. Applied Logic Series, Kluwer Academic Publishers, 2001.

[5] A. Fuhrmann. Theory contraction through base contraction. Journal of Philosophical Logic, 20, 175-203, 1991.

[6] S. O. Hansson. Belief Base Dynamics. PhD thesis, Uppsala University, 1991.

[7] S. O. Hansson. In defense of base contraction. Synthese, 91, 239-245, 1992.

[8] S. O. Hansson. A survey of non-prioritized belief revision. Erkenntnis, 50, 413-427, 1999.

[9] S. O. Hansson. A Textbook of Belief Dynamics. Theory Change and Database Updating. Applied Logic Series. Kluwer Academic Publishers, Dordrecht, 1999.

[10] S. O. Hansson, E. Fermé, J. Cantwell, and M. Falappa. Credibility-limited revision. Journal of Symbolic Logic, 66, 1581-1596, 2001.

[11] D. Makinson. Screened revision. Theoria, 63, 14-23, 1997.

[12] H. Rott. "Just because". Taking belief bases very seriously. In Logic for a Change, S. O. Hansson and W. Rabinowicz, eds, pp. 106-124. Number 9 in Uppsala Prints and Preprints in Philosophy, Deppartment of Philosophy, Uppsala University, 1995.

[13] R. Wassermann. Resource Bounded Belief Revision. PhD thesis, University of Amsterdam, 2000.

[14] M.-A. Williams. On the logic of theory base change. In Logics in Artificial Intelligence, C. MacNish, ed. Number 835 in Lecture Notes in Computer Science. Springer Verlag, 1994.

\section{Appendix}

\section{A Proofs}

\section{Proof of Theorem 3.2}

$(1 \rightarrow 2)$

This proof starts constructing a retractability set $R$ from $\sim$, including in this set every sentence successfully retracted from the belief base $A$ via $\sim$. Then, let $A$ be a belief base and $\sim$ a contraction function that satisfies the properties listed in condition 1 . We define $R$ such that:

$$
R_{\sim}=\{\alpha: A \sim \alpha \not \alpha\} .
$$

We must prove that $R_{\sim}$ satisfies the properties listed in condition 2 .

Nonretractability propagation Let $\alpha$ such that $\alpha \notin R_{\sim}$. Then by definition $A \sim \alpha \vdash \alpha$. Let $\beta$ such that $\alpha \vdash \beta$. By $\sim$ persistence, $A \sim \beta \vdash \alpha$. Supraclassicality yields $A \sim \beta \vdash \beta$. Then $\beta \notin R_{\sim}$.

Nonretractability preservation Let $\beta$, such that $\beta \notin R_{\sim}$ : By definition $R_{\sim}, A \sim \beta \vdash \beta$, then by $\sim$ persistence $A \sim \alpha \vdash \beta$.

Conjunctive completeness Let $\alpha \notin R_{\sim}$ and $\beta \notin R_{\sim}$. It follows by definition that $A \sim \alpha \vdash \alpha$ and $A \sim \beta \vdash \beta$. 


\section{Credibility-limited Functions for Belief Bases}

Then by $\sim$ persistence, $A \sim(\alpha \wedge \beta) \vdash \beta$ and $A \sim(\alpha \wedge \beta) \vdash \alpha$. Then $A \sim(\alpha \wedge \beta) \vdash \alpha \wedge \beta$, from which it follows that $\alpha \wedge \beta \notin R_{\sim}$.

Now we will define a partial meet base contraction function based on $\sim$ and show that it satisfies the equivalence defined in condition 2 . We will first define a selection function based on $\sim$, that allow the construction of a contraction function and satisfies the postulates defined for belief bases (see [6]).

$$
\gamma(A, \alpha)= \begin{cases}A & \text { if } A \perp \alpha=\emptyset \\
A^{\prime} \in A \perp \alpha \text { such that }\left\{\begin{array}{l}
A \sim \alpha \subseteq A^{\prime} \\
A \sim \alpha \vdash \alpha
\end{array} \quad \text { or } \quad\right. \text { otherwise. }\end{cases}
$$

We must prove.

11) $\gamma_{\sim}$ is a well-defined function: in order to $\gamma_{\sim}$ be a function, it must be the case that for all $\alpha_{1}$ and $\alpha_{2}$ if $A \perp \alpha_{1}=A \perp \alpha_{2}$, then $\gamma_{\sim}\left(A, \alpha_{1}\right)=\gamma_{\sim}\left(A, \alpha_{2}\right)$. Suppose that $A \perp \alpha_{1}=A \perp \alpha_{2}$. Then (see [9, obs 1.39 ]) any subset of $A$ implies $\alpha_{1}$ iff implies $\alpha_{2}$. By $\sim$ uniformity, $A \sim \alpha_{1}=A \sim \alpha_{2}$. Hence by definition $\gamma_{\sim}\left(A, \alpha_{1}\right)=\gamma_{\sim}\left(A, \alpha_{2}\right)$.

र2) $\gamma_{\sim}(A, \alpha)=A$ if $A \perp \alpha=\emptyset$ : follows trivially by definition.

子3) $\gamma_{\sim}(A, \alpha) \subseteq A \perp \alpha, \gamma_{\sim}(A, \alpha) \neq \emptyset$ if $A \perp \alpha \neq \emptyset$ : by $\sim$ relative success, $A \sim \alpha=A$ or $A \sim \alpha \forall \alpha$. If $A \sim \alpha \vdash \alpha$ it follows from the definition that $\gamma_{\sim}(A, \alpha)=A \perp \alpha$.

Let $A \sim \alpha \not \alpha$. Then $\forall \alpha$. Then by $\sim$ inclusion $A \sim \alpha \subseteq A$, from which it follows (upper bound property [3] and [9, postulate 1.37]) that there exists $A^{\prime} \in A \perp \alpha$ such that $A \sim \alpha \subseteq A^{\prime}$. Then by definition of $\gamma A^{\prime} \in$ $\gamma_{\sim}(A, \alpha)$.

Due to Theorem 2.3 a partial meet base contraction satisfies success, inclusion, relevance, and uniformity.

$$
A \div \sim \alpha=\bigcap \gamma \sim(A, \alpha)
$$

Finally, we must prove that $A \sim \alpha= \begin{cases}A \div \sim \alpha & \text { if } \alpha \in R_{\sim} \\ A & \text { otherwise. }\end{cases}$

Case 1. $A \sim \alpha \forall \alpha$ : Then by definition, $\alpha \in R_{\sim}$. We want to prove that $A \sim \alpha=A \div \sim \alpha$.

Due to $A \perp \alpha \neq \emptyset$ it follows by definition that $A \sim \alpha \subseteq A^{\prime}$ for all $A^{\prime} \in \gamma_{\sim}(A \perp \alpha)$, hence $A \sim \alpha \subseteq$ $\bigcap(\gamma \sim(A \perp \alpha))=A \div \sim \alpha$.

For the converse, let $\beta \in A \div \sim \alpha$, then by $\div$ inclusion $\beta \in A$. By definition of $\div, \beta \in A^{\prime}$ for all $A^{\prime} \in \gamma_{\sim}(A \perp \alpha)$. Assume by reductio that $\beta \notin A \sim \alpha$. From $\beta \in A$ and $\beta \notin A \sim \alpha$ it follows by $\sim$ relevance that there exists $A^{\prime}$ such that $A \sim \alpha \subseteq A^{\prime}, A^{\prime} \forall \alpha$ and $A^{\prime} \cup\{\beta\} \vdash \alpha$. Then there exists $A^{\prime \prime} \in A \perp \alpha, A^{\prime} \subseteq A^{\prime \prime}$. Since $A^{\prime \prime} \nvdash \alpha$ then $\beta \notin A^{\prime \prime}$, but from definition $\gamma_{\sim}, A^{\prime \prime} \in \gamma_{\sim}(A \perp \alpha)$. Contradiction.

Case 2. $A \sim \alpha \vdash \alpha$ : By vacuity, $A \sim \alpha=A$. By definition $R_{\sim}, \alpha \notin R_{\sim}$. Hence $A \sim \alpha=A$.

$(\mathbf{2} \rightarrow \mathbf{1})$

Let $\div$ be a base contraction function that satisfies success, inclusion, relevance, and uniformity and $R$ a set of sentences that satisfies conjunctive completeness, nonretractability preservation, and non retractability propagation. Let $\sim$ such that:

$$
A \sim \alpha= \begin{cases}A \div \alpha & \text { if } \alpha \in R \\ A & \text { otherwise. }\end{cases}
$$

We must prove that $\sim$ satisfies relative success, inclusion, relevance, persistence, vacuity, and uniformity.

Relative success If $\alpha \notin R$, trivial. Let $\alpha \in R$, then by $\div$ success, $\alpha \notin C n(A \sim \alpha)$ if $\forall \alpha$, in which case (by $\div$ failure) $A \sim \alpha=A$.

Persistence If $\vdash \beta$, trivial. Let $\forall \beta$ and $\beta \in C n(A \sim \beta)$. Then by definition and $\sim$ success $\beta \notin R$, then $\beta \in \mathcal{L} \backslash R$. Hence by nonretractability preservation $\beta \in C n(A \sim \alpha)$.

Inclusion Trivial by definition of $\sim$ and $\div$ inclusion.

Relevance Let $\beta \in A$ and $\beta \notin A \sim \alpha$. Then by $\sim$ relative success, $\alpha \notin C n(A \sim \alpha)$ and by definition $\alpha \in R$. Since $A \sim \alpha \neq A$, it follows by definition that $\sim, A \sim \alpha=A \div \alpha$ and the rest follows by $\div$ relevance.

Vacuity If $\alpha \in R$ follows from definition of $\sim$ and $\div$ vacuity, otherwise, follows from definition of $\sim$.

Uniformity Let be the case that for all subsets $A^{\prime}$ of $A, \alpha \in C n\left(A^{\prime}\right)$ if and only if $\beta \in C n\left(A^{\prime}\right)$. Let $\vdash \alpha$. Then 
$\vdash \beta$. Hence by definition and $\div$ failure $A \sim \alpha=A \sim \beta=A$. Let $\forall \alpha$. Assume without loss of generality that $\alpha \in R$. Then $A \sim \alpha=A \div \alpha$. By $\div$ success $\alpha \notin C n(A \div \alpha)$. By $\div$ uniformity $A \div \alpha=A \div \beta$ and due to $\div$ inclusion $A \div \alpha \subseteq A$. Then by hypothesis $\beta \notin C n(A \div \beta)$, from which it follows by nonretractability preservation that $\beta \in R$. Hence $A \sim \beta=A \div \beta=A \div \alpha=A \sim \alpha$.

Conjunctive constancy Let $A \sim \alpha=A \sim \beta=A$. If $\vdash \alpha$, then by nonretractability propagation $\alpha \wedge \beta \in R$ iff $\beta \in R$, from which it follows by definition and $\div$ uniformity that $A \sim(\alpha \wedge \beta)=A \sim \beta=A$. Idem if $\vdash \beta$. Let $\forall \alpha$ and $\forall \beta$. If $\alpha \notin R$ and $\beta \notin R$, it follows by conjunctive completeness that $\alpha \wedge \beta \notin R$, hence $A \sim(\alpha \wedge \beta)=A$. Assume without loss of generality that $\alpha \in R$. Due to $\div$ success and $\forall \alpha$ it follows that $\alpha \notin C n(A)$, from which it follows that $\alpha \wedge \beta \notin C n A$. Hence, $\div$ vacuity yields $A \sim(\alpha \wedge \beta)=A$

\section{Proof of Theorem 4.3}

$(\mathbf{1} \rightarrow \mathbf{2})$

This proof uses the same schema as the shielded base contraction representation theorem; i.e. we define $C$ induced by $\circledast$ and then we define a selection function based in terms of $\circledast$, which (based on previous results) it is useful to define a contraction function and then, using the Levi identity, arrive at a partial meet base revision function that satisfies the whole AGM postulates.

Let $\circledast$ such that it satisfies the properties listed in condition 1 . We define $C$ such that:

$$
C_{\circledast}=\{\alpha / \alpha \in A \circledast \alpha\} \text {. }
$$

We must prove that $C_{\circledast}$ satisfies the properties listed in condition 2 .

Element consistency Let $\alpha \in C_{\circledast}$. Then $\alpha \in A \circledast \alpha$, from which it follows by $\circledast$ consistency preservation (due to $A \forall \perp$ ) that $A \circledast \alpha \forall \vdash$. Hence supraclassicality yields $\alpha \forall \vdash$.

Single sentence closure Let $\alpha$ and $\beta$ such that $\vdash \alpha \rightarrow \beta$ and $\alpha \in C_{\circledast}$, then by definition of $C_{\circledast}, \alpha \in A \circledast \alpha$. By $\circledast$ strict improvement, $\beta \in A \circledast \beta$. Hence by definition $\beta \in C_{\circledast}$.

Disjunctive completeness Let $(\alpha \vee \beta) \in A \circledast(\alpha \vee \beta)$. It follows by $\circledast$ disjunctive distribution that $\alpha \in A \circledast \alpha$ or $\beta \in A \circledast \beta$, then by definition $\alpha \in C_{\circledast}$ or $\beta \in C_{\circledast}$.

Expansive credibility Let $A \forall \forall$, then by $\circledast$ vacuity $\neg \alpha \in A \circledast \neg \alpha=A \cup\{\neg \alpha\}$, from which it follows by definition of $C_{\circledast}, \neg \alpha \in C_{\circledast}$.

Revision credibility Let $\alpha \in C_{\circledast}$, then by definition of $C_{\circledast}, \alpha \in A \circledast \alpha$. Let $\beta$ such that $A \circledast \alpha \vdash \beta$. $\circledast$ regularity yields $\beta \in A \circledast \beta$. Hence $\beta \in C_{\circledast}$.

Strong revision credibility Let $A \circledast \alpha \forall \neg \beta$. Then by $\circledast$ strong regularity, $\beta \in A \circledast \beta$. Hence $\beta \in C_{\circledast}$.

The next step is to prove that there exists a revision function $*$, that satisfies consistency, success, inclusion, relevance, and uniformity.

We start constructing a selection function based on $\circledast^{3}$ :

$$
\gamma_{\circledast}(A, \alpha)= \begin{cases}\text { A } & \text { If } A \perp \alpha=\emptyset \\
\text { A'}^{\prime} / \mathrm{A}^{\prime} \in \mathrm{A} \perp \alpha \text { and }\{ & \begin{array}{l}
A \cap(A \circledast \neg \alpha) \subseteq A^{\prime} \cup\{\neg \alpha\} \text { or } \\
\neg \alpha \notin A \circledast \neg \alpha
\end{array} \text { otherwise. }\end{cases}
$$

We must prove that:

$\gamma 1) \gamma_{\circledast}$ is a well-defined function: In order to $\gamma_{\circledast}$ be a function, it must be the case that for all $\alpha_{1}$ and $\alpha_{2}$ if $A \perp \alpha_{1}=A \perp \alpha_{2}$, then $\gamma_{\circledast}\left(A, \alpha_{1}\right)=\gamma_{\circledast}\left(A, \alpha_{2}\right)$. Let $A \perp \alpha_{1}=A \perp \alpha_{2}$. Then (see [9, obs. 1.39 ]) any subset of $A$ implies $\alpha_{1}$ iff implies $\alpha_{2}$. Assume without loss of generality that $\neg \alpha_{1} \notin A \circledast \neg \alpha_{1}$. By $\circledast$ relative success, $A \circledast \neg \alpha_{1}=A$, then by $\circledast$ vacuity $A \circledast \neg \alpha_{1} \vdash \alpha_{1}$. Then $A \cap\left(A \circledast \neg \alpha_{1}\right) \vdash \alpha_{1}$, from which it follows by [9, obs. 1.39] that $A \cap\left(A \circledast \neg \alpha_{1}\right) \vdash \alpha_{2}$ and by $\circledast$ uniformity $A \cap\left(A \circledast \neg \alpha_{2}\right) \vdash \alpha_{2}$, from which it follows from $\circledast$ consistency preservation that $\neg \alpha_{2} \notin A \circledast \neg \alpha_{2}$. Hence $\gamma_{\circledast}\left(A, \alpha_{1}\right)=\gamma_{\circledast}\left(A, \alpha_{2}\right)$.

Let $\alpha_{1} \in A \circledast \neg \alpha_{1}$. From [9, obs. 1.39] it follows that any subset of $A$ is inconsistent with $\neg \alpha_{1}$ iff it is inconsistent

\footnotetext{
${ }^{3}$ We will use the same schema as the Representation Theorem 3.6 of [9].
} 


\section{Credibility-limited Functions for Belief Bases}

with $\neg \alpha_{2}$. Then it follows from $\circledast$ uniformity that $A \cap\left(A \circledast \neg \alpha_{1}\right)=A \cap\left(A \circledast \neg \alpha_{2}\right)$. This is sufficient to show that $\gamma_{\circledast}$ is indeed a function.

$\gamma 2) \gamma_{\circledast}(A, a)=A$ if $A \perp \alpha=\emptyset$. Trivial by definition.

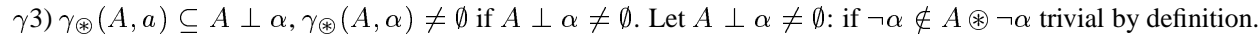
Let $\neg \alpha \in A \circledast \neg \alpha$ : it follows by $A \perp \alpha \neq \emptyset$ that $\forall \alpha$. By $\circledast$ consistency preservation $A \circledast \neg \alpha \forall \alpha$, from which it follows that $A \cap(A \circledast \neg \alpha) \forall \alpha$, then by (upper bound property [3] and [9, postulate 1.37]) there exists $A^{\prime} \in A \perp \alpha$ such that $A \cap(A \circledast \neg \alpha) \subseteq A^{\prime}$, hence $A^{\prime} \in \gamma_{\circledast}(A, \alpha)$.

Due to Theorem 2.3, the function $A \div \circledast \alpha=\bigcap \gamma_{\circledast}(A, a)$ satisfies success, consistency preservation, inclusion, relevance, and uniformity and due to Theorem 2.6, the revision defined by means of the Levi identity $A \star_{\circledast} \alpha=$ $(A \div \circledast \neg \alpha) \cup\{\alpha\}$ satisfies consistency, success, inclusion, relevance, and uniformity.

We will prove that

$$
A \circledast \alpha= \begin{cases}A * \circledast \alpha & \text { if } \alpha \in C_{\circledast} \\ A & \text { otherwise. }\end{cases}
$$

If $\alpha \notin A \circledast \alpha$, by definition of $C_{\circledast}, \alpha \notin C_{\circledast}$. By $\circledast$ relative success $A \circledast \alpha=A$ and we are done.

If $\alpha \in A \circledast \alpha$, then by definition of $C_{\circledast}, \alpha \in C_{\circledast}$. We must prove that $A \circledast \alpha=\bigcap\left(\gamma_{\circledast}(A \perp \neg \alpha)\right) \cup\{\alpha\}$. It follows by $\alpha \in A \circledast \alpha$ and $\circledast$ consistency preservation that $\forall \neg \alpha$. Then $A \perp \neg \alpha \neq \emptyset$, from which it follows by definition of $\gamma_{\circledast}$ and $\alpha \in A \circledast \alpha$ that $A \cap(A \circledast \alpha) \subseteq \bigcap \gamma_{\circledast}(A \perp \neg \alpha)$. Then $(A \cup\{\alpha\}) \cap((A \circledast \alpha) \cup\{\alpha\}) \subseteq \cap\left(\gamma_{\circledast}(A \perp \neg \alpha) \cup\{\alpha\}\right)$. Hence by $\circledast$ inclusion $A \circledast \alpha \subseteq \cap\left(\gamma_{\circledast}(A \perp \neg \alpha)\right) \cup\{\alpha\}$. For the converse we must prove that $\bigcap\left(\gamma_{\circledast}(A \perp \neg \alpha)\right) \cup\{\alpha\} \subseteq A \circledast \alpha$. Since $\alpha \in A \circledast \alpha$, it is suffices to prove that $\bigcap \gamma_{\circledast}(A \perp \neg \alpha) \subseteq A \circledast \alpha$. Let $\beta \in \cap \gamma_{\circledast}(A \perp \neg \alpha)$. Then $\beta \in A^{\prime}$ for all $A^{\prime} \in \gamma_{\circledast}(A \perp \neg \alpha)$. Then $\beta \in A$. Assume by reductio that $\beta \notin A \circledast \alpha$. Then by $\circledast$ relevance there exists $A^{\prime \prime}, A \circledast \alpha \subseteq A^{\prime \prime} \subseteq A \cup\{\alpha\}$ such that $A^{\prime \prime} \forall \perp$, but $A^{\prime \prime} \cup\{\beta\} \vdash \perp$. Then $A^{\prime \prime} \backslash\{a\} \forall \neg \alpha$ and $A^{\prime \prime} \forall \perp$, from which it follows that there exists $A^{\prime \prime \prime} \in A \perp \neg \alpha$ such that $A^{\prime \prime} \subseteq A^{\prime \prime \prime}$. Then $A \circledast \alpha \subseteq A^{\prime \prime} \subseteq A^{\prime \prime \prime} \cup\{\alpha\}$. Definition of $\gamma_{\circledast}$ yields $A^{\prime \prime \prime} \in \gamma_{\circledast}(A \perp \neg \alpha)$. Then $\beta \in A^{\prime \prime \prime}$. Absurd.

$(\mathbf{2} \rightarrow \mathbf{1})$

Let $*$ be a partial meet base revision function, $C$ a set of sentences that satisfies the properties listed in condition 2. Let $\circledast$ be an operator such that:

$$
A \circledast \alpha= \begin{cases}A * \alpha & \text { if } \alpha \in C \\ A & \text { otherwise. }\end{cases}
$$

Relative success trivial by definition and $*$ success.

Consistency preservation Let $A \nvdash \perp$. If $\alpha \in C$ then by element consistency $\forall \neg \alpha$. By definition $A \circledast \alpha=A * \alpha$. Hence, by $*$ consistency, $A \circledast \alpha \not \forall \perp$. If $\alpha \notin C$, trivial.

Inclusion trivial by definition and $*$ inclusion.

Vacuity Let $A \nvdash \neg \alpha$. It follows by expansive credibility that $\alpha \in C$ and the rest follows trivially from $*$ vacuity.

Disjunctive distribution Let $\alpha \notin A \circledast \alpha$ and $\beta \notin A \circledast \beta$. Then by $*$ success $\alpha \notin C$ and $\beta \notin C$. It follows by disjunctive completeness that $(\alpha \vee \beta) \notin C$. Hence $A \circledast(\alpha \vee \beta)=A$. expansive credibility yields $A \vdash \neg(\alpha \vee \beta)$. Hence (since $A \forall \perp),(\alpha \vee \beta) \notin A \circledast(\alpha \vee \beta)$.

Relevance trivial by definition and $*$ relevance.

Strong regularity Let $A \circledast \alpha \not \forall \neg \beta$. Then it follows by strong revision credibility that $\beta \in C$ and the rest follows by $*$ success.

Uniformity Let be the case that for all subsets $A^{\prime} \subseteq A, A^{\prime} \cup\{\alpha\} \vdash \perp$ if and only if $A^{\prime} \cup\{\beta\} \vdash \perp$. If $\neg \alpha \notin C n(A)$, then $\neg \beta \notin C n(A)$. By expansive credibility, $\alpha, \beta \in C$ and the rest follows from $*$ uniformity. Let $\neg \alpha \in C n(A)$, then $\neg \beta \in C n(A)$. Assume without loss of generality that $\alpha \in C$. Then $A \circledast \alpha=A * \alpha$. It follows by element consistency that $\alpha \forall \perp$, then by $*$ consistency $A \circledast \alpha \forall \perp$. By * success, $A \circledast \alpha \forall \neg \alpha$. Then $A \circledast \alpha \cap A \nvdash \neg \alpha$. By hypothesis, $A \circledast \alpha \cap A \forall \neg \beta$. Then $A \circledast \alpha \forall \neg \beta$. By strong revision credibility $\beta \in C$. Hence $\alpha \in C$ iff $\beta \in C$ and the rest follows by $*$ uniformity.

Received 7 May 2002 